Article

\title{
Oral Administration of a Select Mixture of Lactobacillus and Bacillus Alleviates Inflammation and Maintains Mucosal Barrier Integrity in the Ileum of Pigs Challenged with Salmonella Infantis
}

\author{
Xiao Liu, Bing Xia, Ting He, Dan Li, Jin-Hui Su, Liang Guo, Jiu-feng Wang and Yao-Hong Zhu * \\ College of Veterinary Medicine, China Agricultural University, Beijing 100193, China; jizi93112@163.com (X.L.); \\ xiabing26@163.com (B.X.); ting_he413@163.com (T.H.); emma6861@163.com (D.L.); \\ sujh0209@gmail.com (J.-H.S.); binghaitiankong20@163.com (L.G.); jiufeng_wang@hotmail.com (J.-f.W.) \\ * Correspondence: zhu_yaohong@hotmail.com; Tel.: +86-0106-273-1094
}

Received: 25 April 2019; Accepted: 12 May 2019; Published: 15 May 2019

\begin{abstract}
Salmonella is important as both a cause of clinical disease in swine and as a source of food-borne transmission of disease to humans. Lactobacillus and Bacillus are often used as antibiotic substitutes to prevent Salmonella infection. In this study, we evaluated the effects of a select mixture of Lactobacillus johnsonii L531, Bacillus licheniformis BL1721 and Bacillus subtilis BS1715 (LBB-mix) in prevention of Salmonella enterica serovar Infantis infection in a pig model. LBB-mix was orally administered to newly weaned piglets for seven days before $S$. Infantis challenge. LBB-mix pretreatment ameliorated $S$. Infantis-induced fever, leukocytosis, growth performance loss, and ileal inflammation. Pre-administration of LBB-mix reduced the number of Salmonella in the feces but increased the number of goblet cells in the ileum. $S$. Infantis infection resulted in an increase in cell death in the ileum, this increase was attenuated by LBB-mix consumption. Claudin 1 and cleaved caspase- 1 expression was decreased in the ileum of pigs challenged with $S$. Infantis, but not in pigs pretreated with LBB-mix. In conclusion, our data indicate that a select LBB-mix has positive effects on controlling $S$. Infantis infection via alleviating inflammation and maintaining the intestinal mucosal barrier integrity in pigs.
\end{abstract}

Keywords: Lactobacillus; Bacillus; Salmonella Infantis; intestinal mucosal barrier; pig

\section{Introduction}

Salmonella is a common source of food or water-borne infection and causes a wide range of clinical disease in humans and animals. In 2017, a total of 91,662 confirmed human salmonellosis cases were reported in the European Union, and the top five most commonly reported serovars were Salmonella enterica Enteritidis, $S$. Typhimurium, monophasic $S$. Typhimurium, $S$. Infantis and S. Newport [1]. $S$. Infantis causes acute foodborne gastroenteritis, and $S$. Infantis-contaminated pork products are frequent causes of human salmonellosis [2]. The incidence of $S$. Infantis in pigs has increased in recent years [3]. In China, the prevalence of $S$. Infantis in pigs with Salmonella-associated diarrhea was $3.85 \%$ from 2014 to 2016 [4]. Conventional salmonellosis control strategies have relied on antibiotics. However, due to the emergence of drug-resistant strains, many countries have already restricted antibiotic use in animal agriculture. In 2006, the European Union banned the use of antimicrobial growth promoters in animal food and water [5]. In recent years, different intervention strategies such as vaccination, antimicrobial peptides, nutritional supplements, bacteriophages, and probiotics have been used to control Salmonella infection as antibiotic alternatives [6]. 
Different probiotic strains have different effects to prevent or treat diseases. Combined use of probiotic strains has been suggested to have greater efficacy than single strains because they may integrate the effects of different individual strains [7]. Lactobacillus and Bacillus are the most commonly used probiotic agent to improve growth performance, gut health and regulate the immune system in pigs. Our previous study showed that Lactobacillus johnsonii L531 reduced pathogen load and helps maintain short-chain fatty acid levels in the intestines of pigs challenged with S. Infantis [8]. Oral administration of a select mixture of Bacillus probiotics ameliorated Escherichia coli-induced enteritis through preventing loss of intestinal epithelial barrier integrity in weaned pigs [9]. There was evidence that oral supplementation with Bacillus strains increased the abundance of Lactobacillus species in pigs $[10,11]$. Research on the combined use of Lactobacillus and Bacillus to prevent Salmonella infection are still rare.

The intestinal mucosal barrier is the first line of host defense against enteric pathogens, which mainly includes the outer mucus layer, the central single cell layer with specialized epithelial cells, and the inner lamina propria where innate and adaptive immune cells reside. Goblet cells (GCs), known for their apical release of mucin 2 (Muc2), are the predominant secretory epithelial cells lining the luminal surface of the mammalian gastrointestinal tract. Previous studies have shown that oral administration of Lactobacillus acidophilus obviously alleviated S. Typhimurium-induced goblet cells loss in mice [12]. A select mixture of Bacillus licheniformis and Bacillus subtilis ameliorated E. coli-induced decreases in the number of ileal GCs via increasing Atoh1 mRNA expression in newly weaned pigs [10]. Tight junctions (TJs), as a barrier against solute diffusion through the intercellular space, consist mainly of transmembrane protein complexes (e.g., claudin 1 and occludin) and the cytosolic proteins zonula occludins (e.g., ZO-1) [13]. As a crucial component of the epithelial barrier, they are often targeted by pathogenic bacteria, aiding infection and the development of disease [14]. L. acidophilus attenuated Salmonella-induced stress of epithelial cells by modulating TJs genes [15]. B. subtilis significantly enhanced intestinal barrier integrity by up-regulating tight junction protein expression [16].

Salmonella is recognized by the innate immune system and drives pro-inflammatory responses through activation of the inflammatory caspase (caspase- $1,-4,-5$ in humans; and caspase- $1,-11$ in mice). Caspase-1, the most fully characterized inflammatory caspase, cleaves the inactive cytokine precursors pro-interleukin-18 (IL-18) and pro-IL-1 $\beta$, to yield their active secreted forms $[17,18]$. Caspase- 4 is activated when recognizing intracellular lipopolysaccharide [19]. Activated inflammatory caspases also cleave pro-gasdermin-D (GSDMD) and the gasdermin-N domain of GSDMD subsequently forms membrane pores to drive pyroptosis, a lytic form of cell death that triggers inflammation [20-22]. Our previous study demonstrated that pre-incubation with Lactobacillus rhamnosus GR-1 decreased E. coli-induced bovine mammary epithelial cell pyroptosis [23]. However, the role of probiotics in controlling of Salmonella-induced intestinal cell pyroptosis is unclear.

The aim of this study was to determine the ability of a select mixture of Lactobacillus and Bacillus to prevent $S$. Infantis infection and to explore its possible mechanism. We investigated the effects of a select mixture of the potential probiotics Lactobacillus johnsonii L531, Bacillus licheniformis BL1721 and Bacillus subtilis BS1715 (LBB-mix) on S. Infantis infection in newly weaned piglets. We hypothesized that oral administration of LBB-mix alleviates clinical symptoms and inflammation by maintaining the intestinal mucosal barrier integrity and reducing intestinal cell death during $S$. Infantis infection.

\section{Materials and Methods}

\subsection{Bacterial Strains}

A total of 27 Bacillus strains were isolated from the feces collected from 18 healthy newly weaned piglets. Based on the results of in vitro experiments, Bacillus licheniformis BL1721 and Bacillus subtilis BS1715 were selected as putative probiotic strains. Previous studies have demonstrated the probiotic properties of L. johnsonii L531 [8]. 
S. Infantis CAU1508 was isolated from the intestinal contents of weaned pigs with diarrhea, as previously described [24].

\subsection{Characterization of B. licheniformis BL1721 and B. subtilis BS1715 In Vitro}

Characteristics of the two Bacillus isolates were evaluated in comparison to B. licheniformis DSM 5749 and B. subtilis DSM 5750, two strains isolated from an EU-authorized animal probiotic mixture Bioplus ${ }^{\circledR}$ YC (Hørsholm, Denmark).

Tolerance to low $\mathrm{pH}$ and pig bile salts were evaluated by inoculating $10^{8} \mathrm{CFU}$ mid-log phase Bacillus into Luria-Bertani (LB) broth at $\mathrm{pH} 3.0$ or $0.3 \%$ bile salts, respectively. The number of residual viable bacteria was determined at various time points $(0 \mathrm{~h}, 1 \mathrm{~h}, 2 \mathrm{~h}$ and $4 \mathrm{~h})$. Bacteria were diluted appropriately and plated onto LB agar. Plates were incubated at $37^{\circ} \mathrm{C}$ for $24 \mathrm{~h}$, after which the number of $\mathrm{CFU} / \mathrm{mL}$ was calculated.

Bacterial adhesion capacity was assessed using porcine jejunal epithelial J2 cells (IPEC-J2, ACC701, Deutsche Sammlung von Mikroorganismen und Zellkulturen). The IPEC-J2 cells $\left(5 \times 10^{5}\right.$ cells/well $)$ were seeded onto a 6-well trans-well collagen-coated polytetrafluoroethylene (PTFE) filter $(0.4 \mu \mathrm{m}$ pore size, $4.7 \mathrm{~cm}^{2}$ growth area, Corning Costar Corp., Cambridge, MA). Confluent cell monolayers were treated with Bacillus strains $\left(5 \times 10^{7} \mathrm{CFU}\right)$ for $3 \mathrm{~h}, 6 \mathrm{~h}, 9 \mathrm{~h}$, then washed three times with PBS to remove non-adherent bacteria and treated with $0.05 \%$ trypsin for $10 \mathrm{~min}$ at $37^{\circ} \mathrm{C}$. Cells were harvested by centrifugation for $10 \mathrm{~min}$ at $4000 \times g$ and lysed with $100 \mu \mathrm{L}$ of $0.2 \%$ Triton $\mathrm{X}-100$ in sterile water. The populations of Bacillus were determined on LB agar plates.

Trypan blue staining was used to investigate the cell death. The IPEC-J2 cells $\left(5 \times 10^{5}\right.$ cells/well $)$ were seeded onto a 6-well trans-well collagen-coated PTFE filter. Confluent cell monolayers were treated under one of six conditions, as follows: (i) Dulbecco's Modified Eagle's Medium, (Gibco, Grand Island, NY), (ii) B. licheniformis BL1721 ( $\left.5 \times 10^{7} \mathrm{CFU}\right)$, (iii) B. subtilis BS1715 (5 $\left.\times 10^{7} \mathrm{CFU}\right)$, (iv) S. Infantis $\left(5 \times 10^{6} \mathrm{CFU}\right)$, (v) preincubation with B. licheniformis BL1721 $\left(5 \times 10^{7} \mathrm{CFU}\right)$ and exposed to $S$. Infantis $\left(5 \times 10^{6} \mathrm{CFU}\right)$, or (vi) preincubation with B. subtilis BS1715 $\left(5 \times 10^{7} \mathrm{CFU}\right)$ and exposed to $S$. Infantis $\left(5 \times 10^{6} \mathrm{CFU}\right)$. Cells were harvested by digestion with $0.25 \%$ trypsin and centrifugation for $10 \mathrm{~min}$ at $4000 \times \mathrm{g}$. The harvested cells were mixed with $0.4 \%$ Trypan blue dye (Invitrogen, Carlsbad, CA). Counted the blue cells under a microscope. Results are presented as the ratio of the number of dead cells to the total number of cells. Data are presented as the mean \pm standard error of the mean (SEM) of three independent experiments.

\subsection{Ethics Statement}

All experimental animals were treated in accordance with the Guidelines for Laboratory Animal Use and Care from the Chinese Center for Disease Control and Prevention and Rules for Medical Laboratory Animals from the Chinese Ministry of Health, under protocol AW09059102-2, approved by the Animal Ethics Committee of China Agricultural University (approval date: 17 November 2017). All animals used in this study were housed at the experimental facility of the College of Veterinary Medicine, China Agricultural University.

\subsection{Animals}

A total of 24 (Landrace $\times$ Large White) piglets were used in this study. These piglets weaned at 21 days of age and weighed $4.94 \pm 0.18 \mathrm{~kg}$. Prior to the trial, no clinical signs of diarrhea or other diseases were observed in any of the piglets. Feed and water were provided ad libitum.

\subsection{Animal Experiments}

On the day of weaning (day 0 ), the pigs were assigned to three groups ( $n=8$ per group) based on weight. Prior to initiation of the study, all piglets were determined to be free of Salmonella by analysis of feces. Each group received a different treatment, as follows: (i) Control (CN) group, oral administration of $10 \mathrm{~mL}$ sterile physiological saline for 8 days; (ii) S. Infantis (SI) group, oral administration of $10 \mathrm{~mL}$ 
of sterile physiological saline for 7 days and oral challenge with $S$. Infantis on day $8\left(10^{11} \mathrm{CFU} / \mathrm{mL}\right.$, $10 \mathrm{~mL}$ ); (iii) potential probiotic mixture $+S$. Infantis (PS) group, oral administration of $10 \mathrm{~mL}$ potential probiotic mixture (L. johnsonii L531, $10^{8} \mathrm{CFU} / \mathrm{mL}$; B. licheniformis BL1721, $4 \times 10^{5} \mathrm{CFU} / \mathrm{mL}$ and B. subtilis $\left.\mathrm{BS} 1715,4 \times 10^{5} \mathrm{CFU} / \mathrm{mL}\right)$ for 7 days and oral challenge with $S$. Infantis on day $8\left(10^{11} \mathrm{CFU} / \mathrm{mL}, 10 \mathrm{~mL}\right)$. Pigs of each group were housed separately in pens. Feed intake, body weight (BW), clinical symptoms and diarrhea score were recorded every day. On day 13 (5 days post-infection), pigs in these three groups were sacrificed and tissue samples were immediately collected.

\subsection{Clinical Examinations and Microbiological Analyses}

Rectal temperature was measured twice daily, at 7:30 A.M. and 7:30 P.M. respectively. Fecal samples were collected on days $1,4,8(0,6$, and $12 \mathrm{~h}), 9,10,11$ and 12 after weaning for each group. The severity of diarrhea was also scored as previously described [24]. One gram of fresh fecal sample was homogenized in $9 \mathrm{~mL}$ of sterile saline solution, and suitable dilutions of the homogenates were then plated onto selective medium. The groups of fecal microbes studied and the selective mediums (Beijing Land Bridge Technology Co., Beijing, China) employed were as follows: (i) Salmonella, Xylose lysine tergito4 (XLT4) agar; (ii) Lactobacilli, deMan, Rogosa, Sharpe (MRS) agar; (iii) Bacillus spores, Luria-Bertani (LB) agar (inoculated after heat treatment at $80^{\circ} \mathrm{C}$ for $15 \mathrm{~min}$ ); (iv) bifidobacteria, Tryptone Phytone Yeast (TPY) agar; (v) coliforms, eosin-methylene blue (EMB) agar; (vi) enterococci, Pfizer agar. The plates for XLT4 agar, LB agar, EMB agar, and Pfizer agar were incubated for $24 \mathrm{~h}$ at $37{ }^{\circ} \mathrm{C}$ under aerobic conditions, whereas MRS agar, TPY agar plates were incubated under anaerobic conditions for $48 \mathrm{~h}$ at $37^{\circ} \mathrm{C}$. Results were presented as $\log _{10} \mathrm{CFU} / \mathrm{g}$ feces, and all counts were performed in triplicate.

\subsection{Differential Blood Leukocyte Count}

The total white blood cell count was determined using a semiautomated blood cell counter (MEK-6318K; Nihno Kohden, Inc., Tokyo, Japan) and monitored by microscopic differentiation. The proportions of segmented neutrophils, banded neutrophils and lymphocytes were expressed as a percentage of the total number of leukocytes.

\subsection{Histopathologic Scoring}

The proximal, middle, and distal segments (approximately to $10 \times 15 \times 3 \mathrm{~mm}$ ) of the jejunum and ileum were rinsed with sterile physiological saline and then fixed in $4 \%$ paraformaldehyde. Intestinal pathology was evaluated on hematoxylin and eosin-stained jejunal and ileal sections by a single blinded scorer using a validated scoring system [24]. The summation of the scores for each parameter provided an overall inflammation score for each sample, with a range of $0-15$ in the jejunum and 0-18 in the ileum. No less than three separate sections of each sample were examined. Hematoxylin and eosin-stained tissues were visualized and photographed using an Olympus BX41 microscope (Olympus, Tokyo, Japan) equipped with a Canon EOS 550D camera head (Canon, Tokyo, Japan).

\subsection{Alcian Blue/Periodic Acid-Schiff Staining and Subsequent Quantification}

For mucin-containing GC analysis, ileal sections were stained with alcian blue (AB) for acidic mucin at $\mathrm{pH} 2.5$ or periodic acid-Schiff (PAS) stain for neutral mucin detection (Solarbio, Beijing, China), as described by the manufacturer. For quantification of AB/PAS staining, each segment was visualized and photographed using an Olympus BX41 microscope equipped with a Canon EOS 550D camera head. Positively stained GCs and other absorptive epithelial cells were counted in a blind manner in a total of 10 crypts per piglet. Results are presented as the average ratio of GC count to the count of GCs plus other absorptive epithelial cells. 


\subsection{Analysis of DNA Fragmentation}

Intestinal tissue samples were evaluated for DNA fragmentation by terminal deoxynucleotidyl transferase dUTP nick end labeling (TUNEL), employing the In Situ Fluorescein TUNEL Cell Apoptosis Detection Kit (Transgen, Beijing, China). Briefly, intestinal samples were permeabilized with $0.1 \%$ Triton X-100 for $10 \mathrm{~min}$. Subsequently, tissues were incubated with TUNEL reactant at $37^{\circ} \mathrm{C}$ in the dark for $1 \mathrm{~h}$ and washed three times. Sections were examined under an Olympus BX41 microscope equipped with a Canon EOS 550D camera head. Fluorescence intensity was quantified with the Image-Pro Plus 6.0 software (Media Cybernetics, Silver Spring, MD).

\subsection{Western Blotting}

Proteins were extracted from the ileum as previously described [24]. The primary antibodies were as follows: Anti-claudin 1 (1:500 dilution, 13050-1-AP, Proteintech Group, Chicago, IL, USA), anti-occludin (1:1000 dilution, ab31721, Abcam, Cambridge, UK), anti-caspase-1 (1:200 dilution, sc-56036, Santa Cruz Biotechnology, Dallas, TX, USA), anti-caspase-4 (1:1000 dilution, GTX113639, GeneTex, San Antonio, TX, USA), anti-GSDMD (1:500 dilution, sc-81868, Santa Cruz Biotechnology) and anti-Glyceraldehyde-3-phosphate dehydrogenase (GAPDH), (1:1000 dilution, 60004-1-Ig, Proteintech Group). Horseradish peroxidase-conjugated secondary antibodies used were goat anti-mouse IgG (1:5000 dilution, SA00001-1, Proteintech Group) or goat anti-rabbit IgG (1:5000 dilution, SA00001-2, Proteintech Group). The bands were visualized using a Tanon-5200 gel image system (Tanon, Shanghai, China). The intensity of bands was quantified by densitometric analysis using ImageJ software (National Institutes of Health, Bethesda, MD, USA).

\subsection{Statistical Analysis}

Statistical analyses were conducted using SPSS 22.0 software (SPSS Inc., Chicago, IL, USA). For comparisons of the mean values between the three groups, the statistical significance of differences was tested using ANOVA procedures, following Tukey's honestly significant difference post hoc test. Statistical evaluation of the incidence of diarrhea was carried out using Pearson's chi-squared test. GraphPad Prism 7.0 software (GraphPad Software Inc., San Diego, CA, USA) was used to process the data. The data are expressed as the mean \pm SEM. $p$ values: ${ }^{*} p<0.05 ;{ }^{* *} p<0.01 ;{ }^{* * *} p<0.001$.

\section{Results}

\subsection{In Vitro Probiotic Characteristics of B. licheniformis BL1721 and B. subtilis BS1715}

There was no significant difference in the acid and bile salts tolerance between the B. licheniformis BL1721, B. subtilis BS1715, and the two strains isolated from the Bioplus ${ }^{\circledR}$ YC (Figure 1A,B). The number of adhered B. licheniformis BL1721 was $1.31 \times 10^{4} \pm 9.50 \times 10^{2}$ CFU (the mean \pm SEM) at $3 \mathrm{~h}$ after inoculation, and increased to $4.06 \times 10^{4} \pm 5.46 \times 10^{3} \mathrm{CFU}$ at $9 \mathrm{~h}$. The number of B. subtilis BS1715 was $1.13 \times 10^{4} \pm 1.10 \times 10^{3} \mathrm{CFU}$ at $3 \mathrm{~h}$ after inoculation, and increased to $1.86 \times 10^{4} \pm 2.28 \times 10^{3} \mathrm{CFU}$ at $9 \mathrm{~h}$ (Figure 1C). Both isolates and commercial strains have the similar adhesion ability to IPEC-J2 cells. S. Infantis significantly caused IPEC-J2 cell death at $6 \mathrm{~h}$ after infection, and the proportion of dead cells increased with the time of Salmonella infection. Pre-incubation with Bacillus isolates significantly reduced cell death at $12 \mathrm{~h}$ after $S$. Infantis challenge $(p<0.001$ and $p<0.001$, respectively). Cells incubated with Bacillus alone had a low percentage of death (Figure 1D).

\subsection{Clinical Symptoms and Growth Performance}

Before $S$. Infantis challenge, all pigs exhibited a normal rectal temperature $\left(39.22 \pm 0.28^{\circ} \mathrm{C}\right)$. The rectal temperature in the SI group peaked at $24 \mathrm{~h}$ after challenge $\left(40.37 \pm 0.35^{\circ} \mathrm{C}\right)$, and it remained higher until $96 \mathrm{~h}$. Although the rectal temperature in the PS group was higher than the $\mathrm{CN}$ group at $24 \mathrm{~h}\left(40.10 \pm 0.42{ }^{\circ} \mathrm{C}, p=0.001\right)$, there were no differences at other times (Figure 2A). 


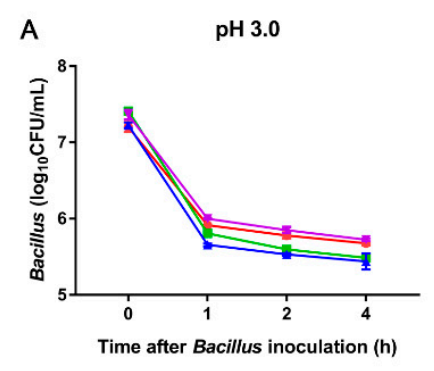

C

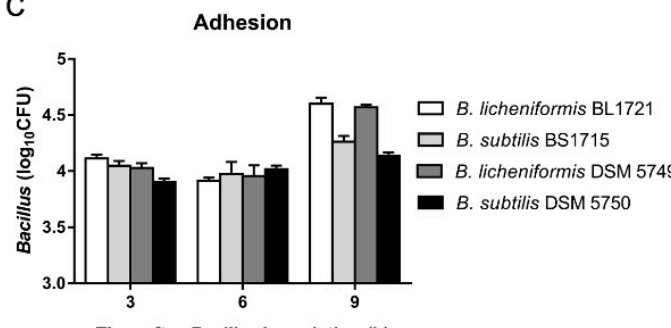

Time after Bacillus inoculation (h)

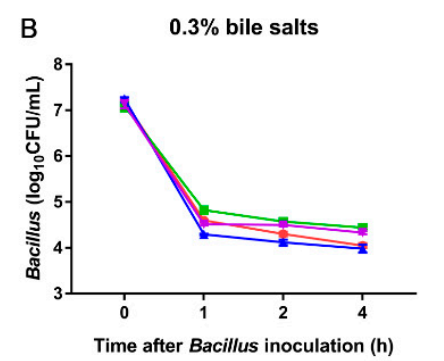

D
- B. licheniformis BL1721

$\rightarrow$ B. subtilis BS1715

- B. licheniformis DSM 5749

* B. subtilis DSM 5750

Figure 1. In vitro assessment of Bacillus for use as livestock probiotics. Survival of Bacillus in $\mathrm{pH} 3.0$ (A) and $0.3 \%$ porcine bile salts (B). (C) The ability of Bacillus to adhere to epithelial cells. (D) Trypan blue staining was used to investigate cell death, the results are presented as the ratio of the number of dead cells to the total number of cells. All analyses were performed in triplicate. ${ }^{*} p<0.05 ;{ }^{* *} p<0.01$; *** $p<0.001$.
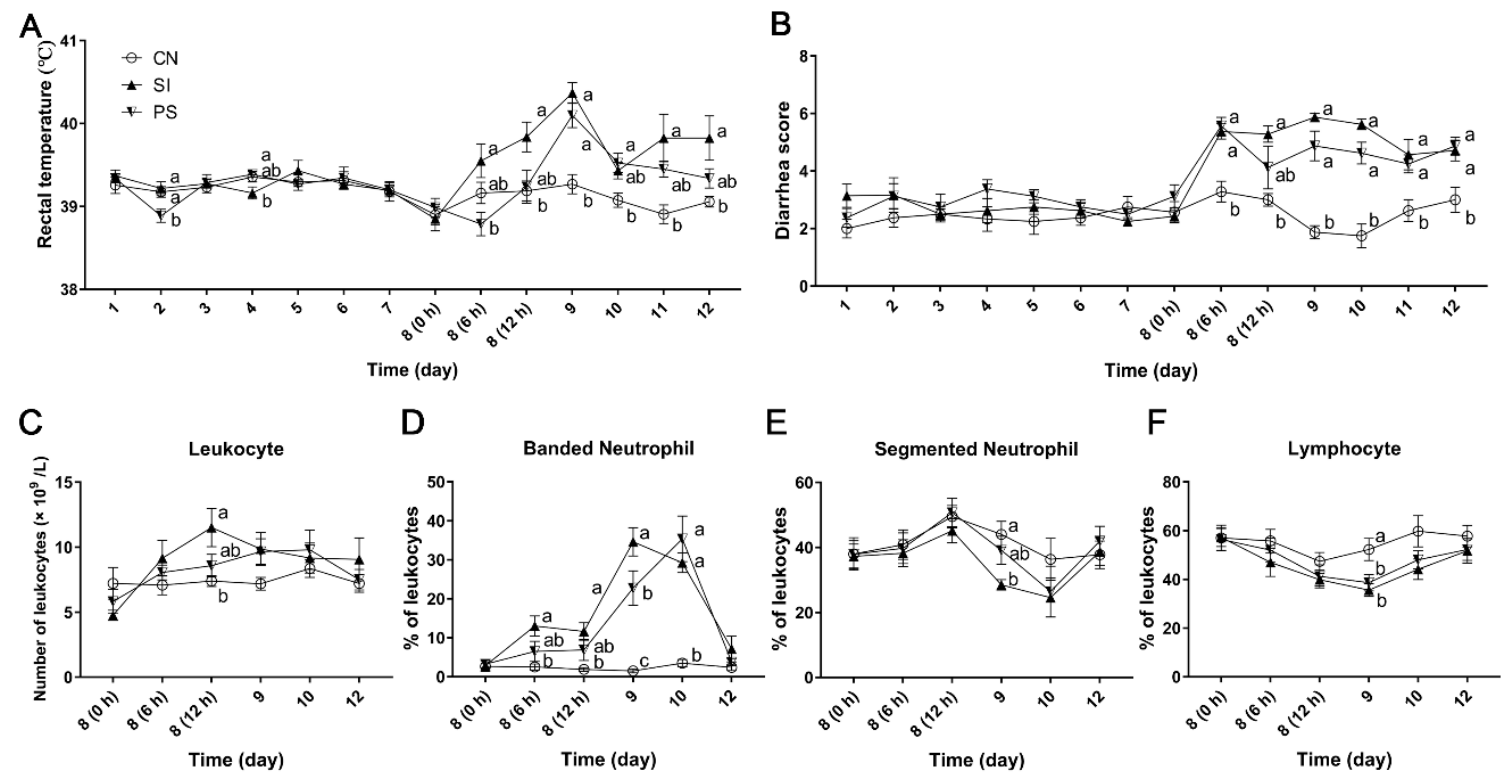

Figure 2. Effects of Lactobacillus johnsonii L531, Bacillus licheniformis BL1721 and Bacillus subtilis BS1715 (LBB-mix) on clinical signs and blood cell differential counts of Salmonella Infantis infection. (A) Rectal temperature and (B) diarrhea scores in pigs ( $n=8$ per group). (C) The total number of peripheral blood leukocytes. The percentage of banded neutrophils (D), segmented neutrophils (E), and lymphocytes (F) on blood leukocytes. Control group with sterile physiological saline $(\mathrm{CN})$; oral sterile physiological saline from day 1 to day 7 followed by $S$. Infantis challenge on day 8 (SI); Pretreated with the potential probiotic mixture for day 1 to day 7 and followed by $S$. Infantis challenge on day 8 (PS). Mean values at the same time point without a common superscript $\left({ }^{a}, b, c\right)$ differ significantly $(p<0.05)$.

Before $S$. Infantis challenge, three groups of pigs had a lower diarrhea rate. During $S$. Infantis infection, the incidence of diarrhea in SI and PS pigs was increased (Table S1). At $6 \mathrm{~h}$ after S. Infantis challenge, all pigs in the SI and PS groups had diarrhea (fecal scores $\geq 4$ ), and this phenomenon 
continued five days after infection (Figure 2B). At $12 \mathrm{~h}$ after challenge, pigs in the SI group, not the PS group, had a higher diarrhea score compared with the $\mathrm{CN}$ group $(p=0.016)$. Although piglets in the SI group had higher scores until $72 \mathrm{~h}$, there was no significant difference between the SI and PS group at other points.

No significant differences were observed in the initial and final body weight between the three groups (Table 1). After S. Infantis challenge, the average daily gain (ADG) of the SI group ( $p=0.002)$ but not the PS group was significantly decreased. Oral challenge with $S$. Infantis also led to a significant reduction in gain to feed ratio (G:F) $(p=0.015)$, whereas administration of the potential probiotic mixture resisted this effect.

Table 1. Effects of oral administration of LBB-mix on the growth performance of weaned piglets before and after $S$. Infantis challenge.

\begin{tabular}{cccccc}
\hline Item ${ }^{1}$ & \multicolumn{3}{c}{ Treatments $^{2}$} & \multirow{2}{*}{ SEM } & \multirow{2}{*}{$p$ Value } \\
\cline { 1 - 4 } & CN & SI & PS & & \\
\cline { 1 - 4 } Pre-challenge (days 1 to 7) & & & & & \\
BW, kg (day 1) & 4.656 & 4.894 & 5.263 & 0.177 & 0.387 \\
ADG, g/d & 136.607 & 143.75 & 149.107 & 10.588 & 0.898 \\
G:F, g/g & 0.615 & 0.701 & 0.612 & 0.048 & 0.707 \\
Post-challenge (days 8 to 13) & & & & & \\
BW, kg (day 13) & 6.3 & 5.85 & 6.443 & 0.211 & 0.379 \\
ADG, g/d & $137.5^{\mathrm{a}}$ & $-10^{\mathrm{b}}$ & $55^{\mathrm{ab}}$ & 19.476 & 0.003 \\
G:F, g/g & $0.495^{\mathrm{a}}$ & $-0.049^{\mathrm{b}}$ & $0.21^{\mathrm{ab}}$ & 0.083 & 0.019 \\
\hline
\end{tabular}

${ }^{1}$ Item: BW, body weight; ADG, average daily gain; G: F, gain to feed ratio; ${ }^{a, b}$ within a row, means without a common lowercase superscript differ $(p<0.05)$; Turkey's test; ${ }^{2}$ Treatments: $\mathrm{CN}$, control group with sterile physiological saline; SI, oral sterile physiological saline from day 1 to day 7 followed by $S$. Infantis challenge; PS, pretreated with potential probiotic mixture for day 1 to day 7 and followed by $S$. Infantis challenge; $n=8$ per group.

\subsection{The Effects of Oral Administration of LBB-Mix on Blood Leukocyte Count and Population Distribution}

Immediately prior to $S$. Infantis challenge, the total number of peripheral blood leukocytes was not significantly different. Compared with the $\mathrm{CN}$ group, an increased peripheral blood leukocyte count was observed at $12 \mathrm{~h}$ after $S$. Infantis challenge in the SI group ( $p=0.025$; Figure $2 \mathrm{C}$ ) but not in the PS group. A continued high percentage of banded neutrophils was observed in the SI group from 6 to $48 \mathrm{~h}$ after challenge $(p=0.007, p=0.008, p<0.001$, and $p<0.001$, respectively; Figure 2D). Although the percentage of banded neutrophils in the PS group increased at $24 \mathrm{~h}$ and $48 \mathrm{~h}$ after challenge $(p=0.001$ and $p<0.001$, respectively), it was lower than the SI group at $24 \mathrm{~h}(p=0.049)$. Compared with the CN group, the SI group exhibited a decrease in the percentage of segmented neutrophils at $24 \mathrm{~h}$ after $S$. Infantis challenge ( $p=0.015$; Figure $2 \mathrm{E})$. We also observed a decrease in the proportion of lymphocytes in the SI and PS groups at $48 \mathrm{~h}$ ( $p=0.010$ and $p=0.036$, respectively; Figure $2 \mathrm{~F})$.

\subsection{The LBB-Mix Changes the Composition of Fecal Microbiota during Salmonella Infection}

The Salmonella detected in the feces of the SI and PS groups persisted from $6 \mathrm{~h}$ to $96 \mathrm{~h}$ after challenge, only one piglet in the PS group was not detected with Salmonella from $72 \mathrm{~h}$. The SI group had a significantly higher Salmonella shedding than the PS group at $6 \mathrm{~h}$ after infection $(p=0.019$; Figure $3 \mathrm{~A})$. After this, the number of Salmonella in the SI group was still higher than that of the PS group, although these differences were not statistically significant.

The number of lactobacilli, Bacillus spores, bifidobacteria, coliforms, and enterococci in the feces were monitored using culture-based enumeration (Figure 3B-F). Prior to $S$. Infantis challenge, on day 1 , fecal enterococci counts were lower in PS pigs than in CN pigs $(p=0.038)$. Pigs in the PS group had higher lactobacilli and bifidobacteria shedding than that in the SI group on day $4(p=0.039$ and $p=0.017$, respectively). After $S$. Infantis challenge, on day 8 , fecal lactobacilli counts were lower in the SI and PS groups than in the $\mathrm{CN}$ group ( $p=0.018$ and $p=0.028$, respectively). On day 11 , fecal enterococci counts were lower in the SI group than in the CN group $(p=0.001)$. Fecal Bacillus spores and coliform populations were unaffected by LBB-mix administration with or without $S$. Infantis challenge. 

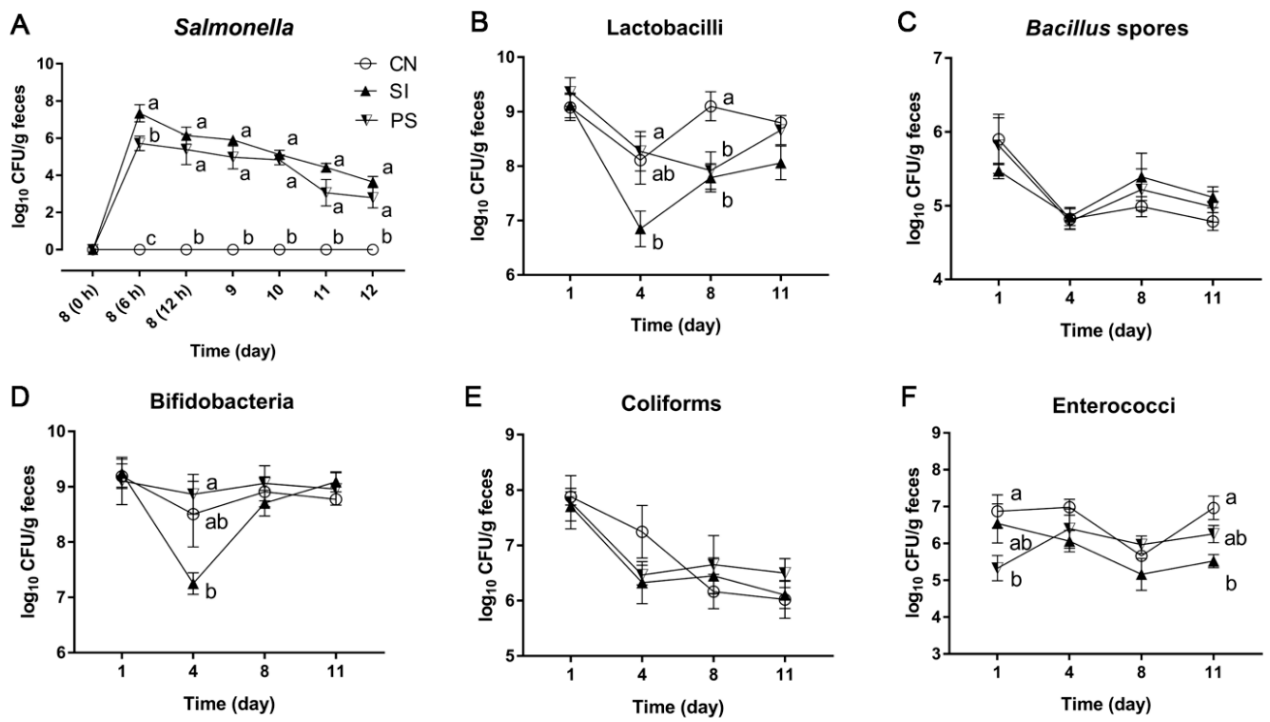

Figure 3. Effects of LBB-mix on the composition of fecal microbiota during $S$. Infantis infection. (A) Salmonella, (B) lactobacilli, (C) Bacillus spores, (D) bifidobacteria, (E) coliforms and (F) enterococci. Fresh fecal samples from all the piglets were collected on days 1, 4, 8 and 11 after weaning. Results are presented as $\log _{10} \mathrm{CFU} / \mathrm{g}$ feces, and all counts were performed in triplicate. Data are presented as the mean $\pm \operatorname{SEM}$ ( $n=8$ per group). Within the same time, mean values with different superscript letters

$\left({ }^{a}, b\right)$ are significantly different $(p<0.05)$.

3.5. Oral Administration of LBB-Mix Attenuated the Severity of Intestinal Damage and Inflammation Induced by S. Infantis

The Salmonella-infected pigs exhibited epithelial cells necrosis and abscission, mucosal hyperemia, submucosa edema, inflammatory cell infiltration, and lymphoid follicle emptying, whereas LBB-mix pretreatment tempered the severity of Salmonella-associated pathological injury and inflammation (Figure 4A). In the jejunal tissue, the histologic score between each group was not significantly different (Figure 4B). In the ileum, the histologic score in the SI group was higher than that in the CN and PS groups ( $p<0.001$ and $p<0.001$, respectively; Figure 4 C). Due to weaning stress, pigs in the CN group experienced slight structural damage.

A

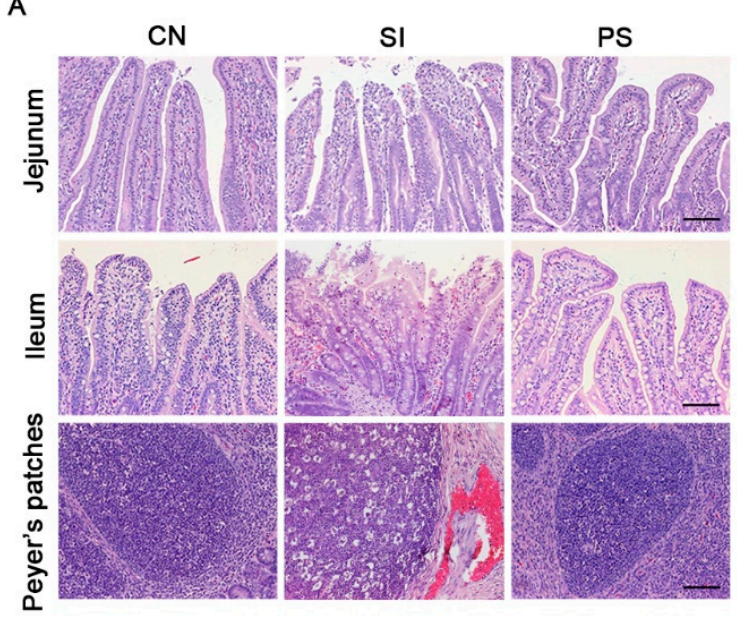

B

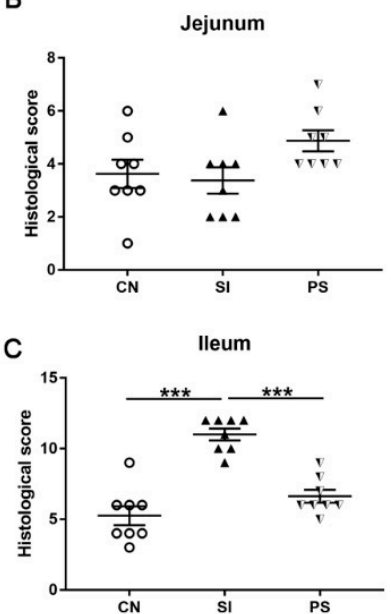

Figure 4. Effects of LBB-mix on the small intestinal inflammation. (A) Representative photomicrographs of hematoxylin and eosin-stained jejunum and ileum sections. Scale bars, $100 \mu \mathrm{m}$. (B,C) Jejunal and ileal histologic scores. Data are presented as the mean $\pm \operatorname{SEM}\left(n=8\right.$ per group). ${ }^{*} p<0.05 ;{ }^{* *} p<0.01$; *** $p<0.001$. 
3.6. Orally Fed LBB-Mix Increased Goblet Cell Count and Claudin 1 Protein Expression during S. Infantis Infection

During infection, the goblet cells in the PS group showed vacuoles containing a large amount of mucus (Figure 5A). There was no difference between $\mathrm{CN}$ and SI pigs with respect to the number of goblet cells in the ileum. However, the number of ileum goblet cells was higher in LBB-mix pretreatment pigs than in $\mathrm{CN}$ pigs $(p=0.011$; Figure $5 \mathrm{~B}$ ). Western blot analysis revealed a reduction in the expression of claudin 1 in the ileum of SI pigs compared with CN pigs ( $p=0.030$; Figure $5 C$ ), pretreatment with LBB-mix can save claudin 1 from its decline. However, there were no differences in the expression of occludin among these three groups (Figure 5D).

A
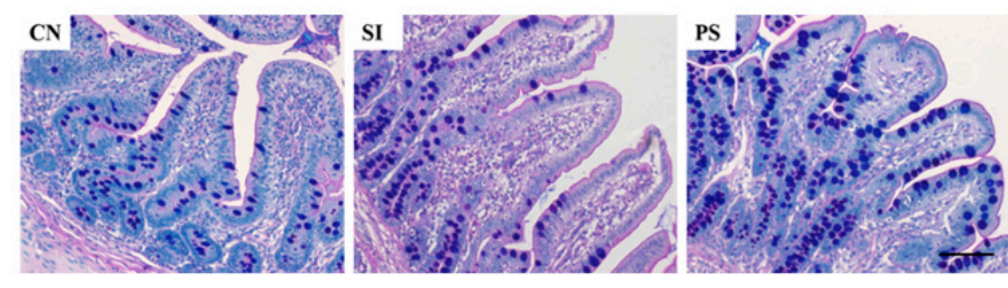

C

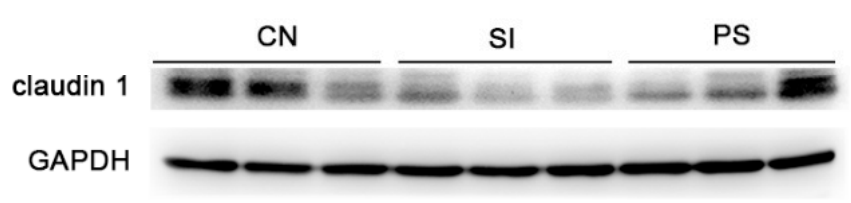

D

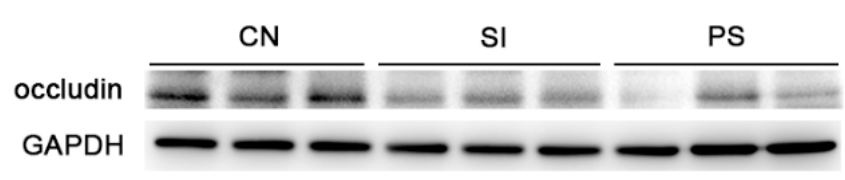

B
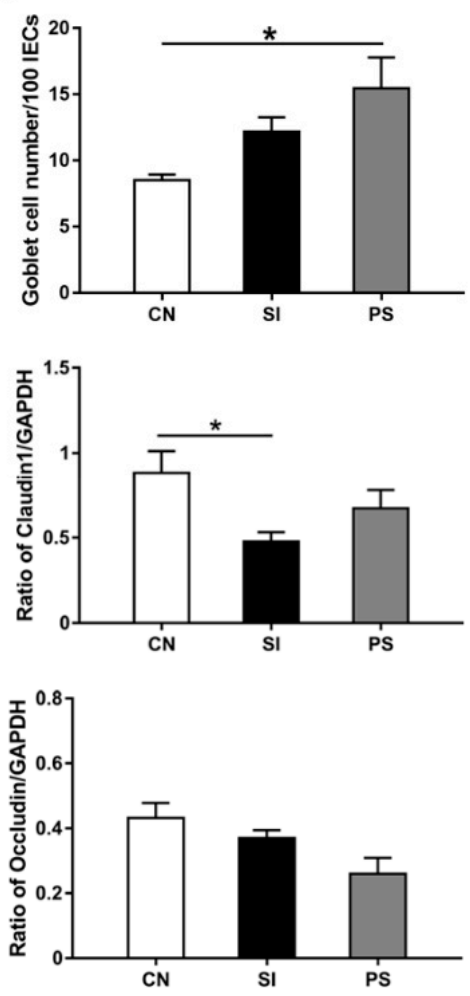

Figure 5. Effects of LBB-mix on the intestinal mucosal barrier in the ileum. (A) Goblet cells in ileal tissues determined by alcian blue/periodic acid-Schiff (AB/PAS) staining, scale bar, $100 \mu \mathrm{m}$. (B) Numbers of goblet cells in the ileum. Representative Western blot results for claudin 1 (C) and occludin (D) in ileal tissues. Each band represents a single pig. Data are presented as the mean $\pm \operatorname{SEM}(n=8$ per group). ${ }^{*} p<0.05 ;{ }^{* *} p<0.01 ; * * * 0.001$.

\subsection{Orally Fed LBB-Mix Reduces Cell Death in the Intestine after S. Infantis Infection}

Compared with the CN group, the DNA damage detected by TUNEL staining in the SI group was upregulated dramatically $(p=0.002$; Figure $6 B)$. Pretreatment with LBB-mix reduced the degree of DNA damage after $S$. Infantis challenge $(p=0.029)$. The expression level of caspase-1 p10 in the SI group was significantly higher than that in the $\mathrm{CN}$ group $(p=0.005$; Figure $6 \mathrm{C})$, and this increase disappeared by oral administration of LBB-mix. Western blot analysis revealed no difference in the expression of cleaved caspase-4 (Figure 6D) and GSDMD-N (Figure 6E) among the three groups. 

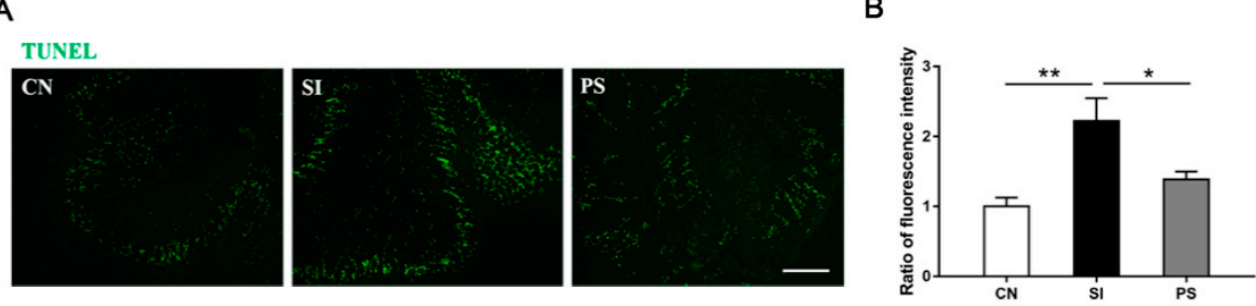

C
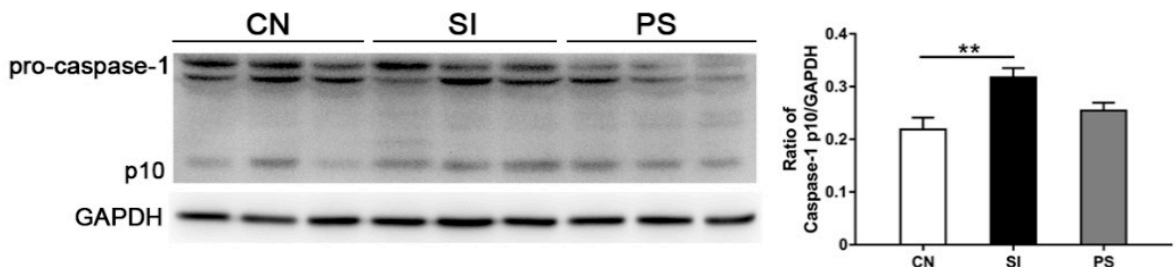

D
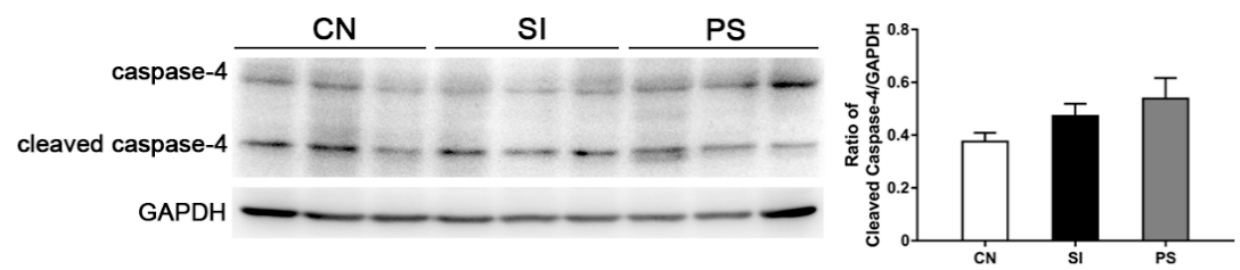

E
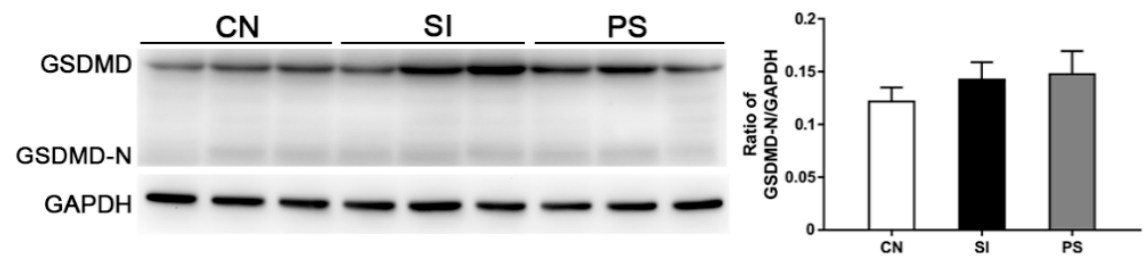

Figure 6. Cell death analysis of the ileum from S. Infantis-infected pigs. (A) Terminal deoxynucleotidyl transferase dUTP nick end labeling (TUNEL) analysis in the ileum. Scale bar, $100 \mu \mathrm{m}$. (B) Quantification of TUNEL fluorescent labeling. Representative Western blot results for caspase-1 (C), caspase-4 (D), and gasdermin-D (GSDMD) (E) in ileal tissues. Each band represents a single pig. Data are presented as the mean $\pm \operatorname{SEM}\left(n=8\right.$ per group). ${ }^{*} p<0.05 ;{ }^{* *} p<0.01 ;{ }^{* * *} p<0.001$.

\section{Discussion}

The potential probiotic mixture used in the present study was composed of L. johnsonii L531, B. licheniforms BL1721 and B. subtilis BS1715, isolated from clinically healthy weaned piglets and selected based on their ability to survive in the simulated gastrointestinal environments and to reduce the cell death caused by Salmonella infection. Bacillus is an aerobic bacterium, which uses oxygen in the intestine, providing an oxygen-free environment for colonization of Lactobacillus. The present study showed that newly weaned pigs challenged with $S$. Infantis were particularly susceptible, and the sequence of clinical symptoms was generally diarrhea, fever, anorexia, depression, and weight loss. $S$. Infantis caused significant intestinal inflammation in the ileum but not in the jejunum, and oral administration of LBB-mix to pigs could ameliorate ileitis caused by $S$. Infantis infection.

Pathogenic microorganisms such as Salmonella have evolved intricate strategies to overcome or manipulate intestinal barrier integrity. This leads to translocation of the pathogen into the lamina propria facilitating further infection of the host and inducing salmonellosis. As an important part of the intestinal barrier, goblet cells secrete mucus, trefoil peptides, and resistin-like molecule- $\beta$, which are central to both the defense and repair of the epithelial layer and have significant roles in epithelial homeostasis [25]. Early study has shown that $S$. Typhimurium infection reduces the number of goblet cells in the cecum of the streptomycin-pretreated mice [26]. We did not observe this phenomenon in piglets infected with $S$. Infantis. The LBB-mix administration increased the number of goblet 
cells during $S$. Infantis infection, thereby increasing the total amount of goblet cell mucin secretion, which not only facilitates the expulsion of Salmonella but also maintains the integrity of the mucus layer.

In fact, one of the effects of probiotics exert is to improve the intestinal barrier integrity by strengthening the apical junction complexes of enterocytes and restoring the structures of microfilaments extending into the terminal web. L. reuteri 15007 significantly enhanced the protein levels of intestinal epithelial claudin 1, occludin and ZO-1 in newly weaned pigs [27]. Our data indicate that LBB-mix may enhance intestinal epithelial barrier integrity through upregulation of claudin 1 but not occludin. Paradoxically, a previous study showed that Lactobacillus- and Bacillus-based probiotics obviously increased the protein level of occludin [28], which might be due to different experimental conditions including probiotic strains and animals. Actually, the role of occludin in the assembly and maintenance of TJs is not only related to its expression, but also to different modification events [29].

A complete intestinal barrier reduces Salmonella invasion and protects intestinal cells from excessive death. In our study, an increase of TUNEL positive labeling was observed in the ileum of pigs infected with $S$. Infantis only, but not in pigs pretreated with the probiotic mixture. Both apoptotic and pyroptotic cells showed positive TUNEL staining. However, unlike apoptosis, which is generally considered to be non-inflammatory, pyroptosis is a highly inflammatory form of programmed cell death. There is evidence that Salmonella infection causes pyroptosis in porcine mesenteric lymph nodes [30]. Since significant ileal inflammation was observed in the SI group, we propose that the infected cells undergo pyroptosis in the ileum, and the probiotic mixture pretreatment can attenuate cell pyroptosis thus prevent damage caused by Salmonella.

Caspase- 1 is the best-characterized inflammatory caspase and is the central effector protein of the inflammasome. Previous studies indicate that Salmonella activates the NLRP3 and NLRC4 inflammasome, resulting in caspase- 1 activation and rapid cell death [31,32]. There was evidence that L. johnsonii N6.2 suppressed the inflammasome and caspase-1 maturation lowering overall gastro-intestinal inflammation [33]. In our study, increased maturation of caspase-1 was observed in the SI but not in the PS piglets. This result indicated that the canonical inflammasome pathway was activated in the ileum of Salmonella-infected pigs, and the probiotic mixture pretreatment can reduce excessive activation of caspase- 1 thus prevent intestinal inflammation caused by Salmonella infection.

Various studies have investigated the potential of caspase- 4 to initiate innate immune responses to intracellular Salmonella infection or Salmonella-derived pathogen associated molecular patterns [34,35]. It remains unclear whether porcine caspase-4 represents functional orthologs of human caspase-4, -5 or murine caspase-11. We examined the expression of caspase- 4 in the ileum of pigs, but there was no difference in cleaved caspase- 4 among the three groups, whether caspase- 4 can play a role in the infection of Salmonella in pigs remains to be further explored.

GSDMD is an executioner of pyroptosis owing to its ability to be cleaved by inflammatory caspases and its ability to form membrane pores. Either caspase- 1 or caspase- 4 independently cleaves GSDMD, from which the released amino-terminal fragment associates with the cell membrane and oligomerizes to form the pyroptotic pore. The cell then swells, resulting in membrane rupture that is known as pyroptosis. Triggering pyroptosis is a property shared by multiple S. enterica serovars. Unexpectedly, in our study, we did not observe the obvious cleavage GSDMD in the ileum of Salmonella-infected pigs, and the Western blot analysis revealed no difference in the expression of GSDMD-N among the three groups. It is probably because pyroptosis is a relatively early event and our sampling time is later. Salmonella can evade the host immune system by down-regulating the expression of PrgJ or flagellin. Previous studies in mice have suggested that $S$. Typhimurium efficiently evade inflammasomes during the systemic phase of infection [36,37]. Another possible explanation is that other Gasdermin family members induced pyroptosis in the ileum of pigs infected with Salmonella [38].

\section{Conclusions}

In conclusion, our data suggest that a select mixture of Lactobacillus and Bacillus is effective in preventing Salmonella infection in newly weaned pigs by maintaining the intestinal mucosal barrier 
integrity and reducing intestinal cell death, although it does not prevent diarrhea. Our findings provide a new preventative strategy that can ameliorate $S$. Infantis-induced ileal inflammation in pigs and thus help to reduce the use of antibiotics. However, the differences in the effects between probiotic mixture and single strains were not compared in the current study. More research is still needed to obtain symbiotic or synergistic combinations to maximize the positive benefits of these probiotic combinations.

Supplementary Materials: The following are available online at http://www.mdpi.com/2076-2607/7/5/135/s1, Table S1: Effects of oral administration of LBB-mix on the incidence of diarrhea in newly weaned pigs before and after $S$. Infantis challenge.

Author Contributions: X.L. participated in the study design, performed the experiments, analyzed the data, and wrote the manuscript; B.X. performed the histologic assessment; T.H. performed bacterial isolation and preparation; D.L. performed the blood leukocyte count; J.-H.S. and L.G. performed the clinical examinations; J.-f.W. and Y.-H.Z. conceived and designed the study.

Funding: This work was funded by the National Key R\&D Program of China (grant number 2017YFD0502200), the National Natural Science Foundation of China (grant numbers 31672613 and 31873034).

Acknowledgments: We thank Wei Zhang and Guiyan Yang for editing the manuscript.

Conflicts of Interest: The authors declare no conflict of interest.

\section{References}

1. European Food Safety Authority and European Centre for Disease Prevention and Control (EFSA and ECDC). The European Union summary report on trends and sources of zoonoses, zoonotic agents and food-borne outbreaks in 2017. EFSA J. 2018, 16, e05500.

2. Schroeder, S.; Harries, M.; Prager, R.; Höfig, A.; Ahrens, B.; Hoffmann, L.; Rabsch, W.; Mertens, E.; Rimek, D. A prolonged outbreak of Salmonella Infantis associated with pork products in central Germany, April-October 2013. Epidemiol. Infect. 2016, 144, 1429-1439. [CrossRef] [PubMed]

3. Yuan, C.; Krull, A.; Wang, C.; Erdman, M.; Fedorka-Cray, P.; Logue, C.; O'Connor, A.M. Changes in the prevalence of Salmonella serovars associated swine production and correlations of avian, bovine and swine-associated serovars with human-associated serovars in the United States (1997-2015). Zoonoses Public Health 2018, 65, 648-661. [CrossRef] [PubMed]

4. Su, J.-H.; Zhu, Y.-H.; Ren, T.-Y.; Guo, L.; Yang, G.-Y.; Jiao, L.-G.; Wang, J.-F. Distribution and Antimicrobial Resistance of Salmonella Isolated from Pigs with Diarrhea in China. Microorganisms 2018, 6, 117. [CrossRef] [PubMed]

5. Van Boeckel, T.P.; Brower, C.; Gilbert, M.; Grenfell, B.T.; Levin, S.A.; Robinson, T.P.; Teillant, A.; Laxminarayan, R. Global trends in antimicrobial use in food animals. Proc. Natl. Acad. Sci. USA 2015, 112, 5649-5654. [CrossRef] [PubMed]

6. Wilhelm, B.J.; Young, I.; Cahill, S.; Nakagawa, R.; Desmarchelier, P.; Rajić, A. Rapid systematic review and meta-analysis of the evidence for effectiveness of primary production interventions to control Salmonella in beef and pork. Prev. Vet. Med. 2017, 147, 213-225. [CrossRef] [PubMed]

7. Chapman, C.; Gibson, G.R.; Rowland, I. Health benefits of probiotics: Are mixtures more effective than single strains? Eur. J. Nutr. 2011, 50,1-17. [CrossRef] [PubMed]

8. He, T.; Zhu, Y.-H.; Yu, J.; Xia, B.; Liu, X.; Yang, G.-Y.; Su, J.-H.; Guo, L.; Wang, M.-L.; Wang, J.-F. Lactobacillus johnsonii L531 reduces pathogen load and helps maintain short-chain fatty acid levels in the intestines of pigs challenged with Salmonella enterica Infantis. Vet. Microbiol. 2019, 230, 187-194. [CrossRef]

9. Yang, G.-Y.; Zhu, Y.-H.; Zhang, W.; Zhou, D.; Zhai, C.-C.; Wang, J.-F. Influence of orally fed a select mixture of Bacillus probiotics on intestinal T-cell migration in weaned MUC4 resistant pigs following Escherichia coli challenge. Vet. Res. 2016, 47, 71. [CrossRef]

10. Zhang, W.; Zhu, Y.-H.; Zhou, D.; Wu, Q.; Song, D.; Dicksved, J.; Wang, J.-F. Oral administration of a select mixture of Bacillus probiotics affects the gut microbiota and goblet cell function following Escherichia coli challenge in newly weaned pigs of genotype MUC4 that are supposed to be enterotoxigenic E. coli F4ab/ac receptor negative. Appl. Environ. Microbiol. 2017, 83, e02747-16. [CrossRef] [PubMed] 
11. Baker, A.; Davis, E.; Spencer, J.; Moser, R.; Rehberger, T. The effect of a Bacillus-based direct-fed microbial supplemented to sows on the gastrointestinal microbiota of their neonatal piglets. J. Anim. Sci. 2013, 91, 3390-3399. [CrossRef] [PubMed]

12. Wu, H.; Ye, L.; Lu, X.; Xie, S.; Yang, Q.; Yu, Q. Lactobacillus acidophilus Alleviated Salmonella-Induced Goblet Cells Loss and Colitis by Notch Pathway. Mol. Nutr. Food Res. 2018, 62, 1800552. [CrossRef] [PubMed]

13. Groschwitz, K.R.; Hogan, S.P. Intestinal barrier function: Molecular regulation and disease pathogenesis. J. Allergy Clin. Immunol. 2009, 124, 3-20. [CrossRef] [PubMed]

14. Landy, J.; Ronde, E.; English, N.; Clark, S.K.; Hart, A.L.; Knight, S.C.; Ciclitira, P.J.; Al-Hassi, H.O. Tight junctions in inflammatory bowel diseases and inflammatory bowel disease associated colorectal cancer. World J. Gastroenterol. 2016, 22, 3117. [CrossRef]

15. Lépine, A.F.; de Wit, N.; Oosterink, E.; Wichers, H.; Mes, J.; de Vos, P. Lactobacillus acidophilus attenuates Salmonella-induced stress of epithelial cells by modulating tight-junction genes and cytokine responses. Front. Microbiol. 2018, 9. [CrossRef]

16. Rhayat, L.; Maresca, M.; Nicoletti, C.; Perrier, J.; Brinch, K.S.; Christian, S.; Devillard, E.; Eckhardt, E. Effect of Bacillus subtilis strains on intestinal barrier function and inflammatory response. Front. Immunol. 2019, 10. [CrossRef] [PubMed]

17. Shalini, S.; Dorstyn, L.; Dawar, S.; Kumar, S. Old, new and emerging functions of caspases. Cell Death Differ. 2015, 22, 526. [CrossRef]

18. Tapia, V.S.; Daniels, M.J.; Palazón-Riquelme, P.; Dewhurst, M.; Luheshi, N.M.; Rivers-Auty, J.; Green, J.; Redondo-Castro, E.; Kaldis, P.; Lopez-Castejon, G. The three cytokines IL-1 $\beta$, IL-18, and IL-1 $\alpha$ share related but distinct secretory routes. J. Biol. Chem. 2019. [CrossRef]

19. Shi, J.; Zhao, Y.; Wang, Y.; Gao, W.; Ding, J.; Li, P.; Hu, L.; Shao, F. Inflammatory caspases are innate immune receptors for intracellular LPS. Nature 2014, 514, 187. [CrossRef] [PubMed]

20. Shi, J.; Zhao, Y.; Wang, K.; Shi, X.; Wang, Y.; Huang, H.; Zhuang, Y.; Cai, T.; Wang, F.; Shao, F. Cleavage of GSDMD by inflammatory caspases determines pyroptotic cell death. Nature 2015, 526, 660. [CrossRef]

21. Kayagaki, N.; Stowe, I.B.; Lee, B.L.; O’Rourke, K.; Anderson, K.; Warming, S.; Cuellar, T.; Haley, B.; Roose-Girma, M.; Phung, Q.T. Caspase-11 cleaves gasdermin D for non-canonical inflammasome signalling. Nature 2015, 526, 666. [CrossRef]

22. He, W.-T.; Wan, H.; Hu, L.; Chen, P.; Wang, X.; Huang, Z.; Yang, Z.-H.; Zhong, C.-Q.; Han, J. Gasdermin D is an executor of pyroptosis and required for interleukin-1 $\beta$ secretion. Cell Res. 2015, 25, 1285. [CrossRef]

23. Wu, Q.; Zhu, Y.-H.; Xu, J.; Liu, X.; Duan, C.; Wang, M.-J.; Wang, J.-F. Lactobacillus rhamnosus GR-1 Ameliorates Escherichia coli-induced Activation of NLRP3 and NLRC4 Inflammasomes with Differential Requirement for ASC. Front. Microbiol. 2018, 9. [CrossRef] [PubMed]

24. Yang, G.-Y.; Yu, J.; Su, J.-H.; Jiao, L.-G.; Liu, X.; Zhu, Y.-H. Oral administration of Lactobacillus rhamnosus GG ameliorates Salmonella Infantis-induced inflammation in a pig model via activation of the IL-22BP/IL-22/STAT3 pathway. Front. Cell. Infect. Microbiol. 2017, 7, 323. [CrossRef] [PubMed]

25. Birchenough, G.M.; Johansson, M.E.; Gustafsson, J.K.; Bergström, J.H.; Hansson, G.C. New developments in goblet cell mucus secretion and function. Mucosal Immunol. 2015, 8, 712. [CrossRef] [PubMed]

26. Barthel, M.; Hapfelmeier, S.; Quintanilla-Martínez, L.; Kremer, M.; Rohde, M.; Hogardt, M.; Pfeffer, K.; Rüssmann, H.; Hardt, W.-D. Pretreatment of mice with streptomycin provides a Salmonella enterica serovar Typhimurium colitis model that allows analysis of both pathogen and host. Infect. Immunity 2003, 71, 2839-2858. [CrossRef]

27. Yang, F.; Wang, A.; Zeng, X.; Hou, C.; Liu, H.; Qiao, S. Lactobacillus reuteri 15007 modulates tight junction protein expression in IPEC-J2 cells with LPS stimulation and in newborn piglets under normal conditions. BMC Microbiol. 2015, 15, 32. [CrossRef] [PubMed]

28. Song, J.; Xiao, K.; Ke, Y.; Jiao, L.; Hu, C.; Diao, Q.; Shi, B.; Zou, X. Effect of a probiotic mixture on intestinal microflora, morphology, and barrier integrity of broilers subjected to heat stress. Poult. Sci. 2014, 93, 581-588. [CrossRef]

29. Cummins, P.M. Occludin: One protein, many forms. Mol. Cell. Biol. 2012, 32, 242-250. [CrossRef]

30. Martins, R.P.; Aguilar, C.; Graham, J.E.; Carvajal, A.; Bautista, R.; Claros, M.G.; Garrido, J.J. Pyroptosis and adaptive immunity mechanisms are promptly engendered in mesenteric lymph-nodes during pig infections with Salmonella enterica serovar Typhimurium. Vet. Res. 2013, 44, 120. [CrossRef] [PubMed] 
31. Mariathasan, S.; Newton, K.; Monack, D.M.; Vucic, D.; French, D.M.; Lee, W.P.; Roose-Girma, M.; Erickson, S.; Dixit, V.M. Differential activation of the inflammasome by caspase-1 adaptors ASC and IPAF. Nature 2004, 430, 213. [CrossRef] [PubMed]

32. Broz, P.; Newton, K.; Lamkanfi, M.; Mariathasan, S.; Dixit, V.M.; Monack, D.M. Redundant roles for inflammasome receptors NLRP3 and NLRC4 in host defense against Salmonella. J. Exp. Med. 2010, 207, 1745-1755. [CrossRef] [PubMed]

33. Teixeira, L.; Kling, D.; Lorca, G.; Gonzalez, C. Lactobacillus johnsonii N6. 2 diminishes caspase-1 maturation in the gastrointestinal system of diabetes prone rats. Benef. Microbes 2018, 9, 527-539. [CrossRef] [PubMed]

34. Knodler, L.A.; Crowley, S.M.; Sham, H.P.; Yang, H.; Wrande, M.; Ma, C.; Ernst, R.K.; Steele-Mortimer, O.; Celli, J.; Vallance, B.A. Noncanonical inflammasome activation of caspase-4/caspase-11 mediates epithelial defenses against enteric bacterial pathogens. Cell Host Microbe 2014, 16, 249-256. [CrossRef] [PubMed]

35. Baker, P.J.; Boucher, D.; Bierschenk, D.; Tebartz, C.; Whitney, P.G.; D'Silva, D.B.; Tanzer, M.C.; Monteleone, M.; Robertson, A.A.; Cooper, M.A. NLRP3 inflammasome activation downstream of cytoplasmic LPS recognition by both caspase-4 and caspase-5. Eur. J. Immunol. 2015, 45, 2918-2926. [CrossRef] [PubMed]

36. Miao, E.A.; Mao, D.P.; Yudkovsky, N.; Bonneau, R.; Lorang, C.G.; Warren, S.E.; Leaf, I.A.; Aderem, A. Innate immune detection of the type III secretion apparatus through the NLRC4 inflammasome. Proc. Natl. Acad. Sci. USA 2010, 107, 3076-3080. [CrossRef] [PubMed]

37. Miao, E.A.; Leaf, I.A.; Treuting, P.M.; Mao, D.P.; Dors, M.; Sarkar, A.; Warren, S.E.; Wewers, M.D.; Aderem, A. Caspase-1-induced pyroptosis is an innate immune effector mechanism against intracellular bacteria. Nat. Immunol. 2010, 11, 1136. [CrossRef] [PubMed]

38. Ding, J.; Wang, K.; Liu, W.; She, Y.; Sun, Q.; Shi, J.; Sun, H.; Wang, D.-C.; Shao, F. Pore-forming activity and structural autoinhibition of the gasdermin family. Nature 2016, 535, 111. [CrossRef] [PubMed]

(C) 2019 by the authors. Licensee MDPI, Basel, Switzerland. This article is an open access article distributed under the terms and conditions of the Creative Commons Attribution (CC BY) license (http://creativecommons.org/licenses/by/4.0/). 